\title{
Glucose Transport and Microvillus Membrane Physical Properties along the Crypt-Villus Axis of the Rabbit
}

\author{
J. B. Meddings, D. DeSouza, M. Goel, and S. Thiesen \\ Gastrointestinal Research Group, University of Calgary, Calgary, Alberta T2N 4NI, Canada
}

\begin{abstract}
Both transport function and microvillus membrane physical properties evolve as the enterocyte matures and migrates up the crypt-villus axis. We isolated enriched fractions of villus tip, mid-villus, and crypt enterocytes from which microvillus membrane vesicles were prepared. Using this material we characterized the alterations that occur in microvillus membrane fluidity as the rabbit enterocyte matures and correlated these with kinetic studies of glucose transport. With increasing maturity the microvillus membrane becomes more rigid due to both an increase in the cholesterol/phospholipid ratio and alterations in individual phospholipid subclasses. Maximal rates of glucose transport were greatest in microvillus membrane vesicles prepared from mature cells. However, the glucose concentration producing half-maximal rates of transport $\left(K_{m}\right)$ was significantly lower in crypt microvillus membrane vesicles, suggesting that a distinct glucose transporter existed in crypt enterocytes. This distinction disappeared when differences between membrane lipid environments were removed. By fluidizing villus-tip microvillus membrane vesicles, in vitro, to levels seen in the crypt microvillus membrane, we observed a reduction in the $K_{\mathrm{m}}$ of this transport system. These data suggest that the kinetic characteristics of the sodium-dependent glucose transporter are dependent upon its local membrane environment. (J. Clin. Invest. 1990. 85:1099-1107.) membrane fluidity $\bullet$ nutrient absorption • transport kinetics
\end{abstract}

\section{Introduction}

The intestinal epithelium is a dynamic structure that undergoes constant regeneration. Over a $\sim 3-d$ cycle, enterocytes are formed in the intestinal crypts, migrate up the villus, and are finally sloughed from the villus tips into the intestinal stream. Therefore, at any instant in time enterocytes along the crypt-villus axis represent all stages in the normal, physiological development of the enterocyte. Over this spectrum, marked functional changes are known to exist. Immature enterocytes, found in the crypts, are predominantly secretory, lack disaccharidase enzymes, and have transport systems different from those observed in more mature enterocytes. Furthermore, recent studies in the rat have suggested that the

Address reprint requests to Dr. J. Meddings, Department of Internal Medicine, University of Calgary, 3330 Hospital Drive N.W., Calgary, Alberta T2N 4N1, Canada.

Received for publication 27 March 1989 and in revised form 1 November 1989.

J. Clin. Invest.

(C) The American Society for Clinical Investigation, Inc.

0021-9738/90/04/1099/09 \$2.00

Volume 85, April 1990, 1099-1107 microvillus membrane undergoes a maturational process in terms of its physical properties as the enterocyte migrates up the villus (1). This membrane was found to be more fluid in the crypt cell than in mature cells isolated from the villus tips. Since membrane physical properties can alter the functional activity of transporters embedded within the membrane, it is unclear whether the differences in physiological function observed between crypt and villus-tip cells are due to different protein transporters or whether membrane physical properties play a role.

The present study was designed to address three key issues. First, we wished to confirm earlier studies suggesting that the microvillus membrane becomes more rigid as the enterocyte matures, and if so by what underlying mechanism. Secondly, is the reduced ability of the immature enterocyte to absorb glucose accompanied by a reduction in the rate of glucose transport across the microvillus membrane? Thirdly, do the kinetic characteristics of sodium-dependent glucose absorption change with maturity; i.e., are the glucose transporters found in crypt microvillus membrane different from those present in villus-tip cells, and if so are these alterations related to membrane physical properties?

\section{Methods}

All chemicals used in this study were obtained from either Sigma Chemical Co. (St. Louis, MO) or Fisher Scientific Co. (Pittsburgh, PA) and were of the highest grade available. $\left[{ }^{3} \mathrm{H}\right]$ Glucose and $\left[{ }^{14} \mathrm{C}\right]$ thymidine were obtained from New England Nuclear (Boston, MA) and used as supplied. Fluorescent probes were purchased from Molecular Probes Inc. (Junction City, OR) and also used as supplied.

Statistical analysis was performed using the statistical software Systat (Evanston, IL). Differences between means were evaluated by analysis of variance with specific differences tested using the Tukey analysis as a post hoc test. Curve fitting and parameter estimation for nonlinear relationships were performed with data weighted in proportion to the inverse of within sample variance. Statistical differences between parameter estimates were evaluated using recently described techniques (2).

Cell and membrane isolation. Animals used for these studies were male New Zealand white rabbits weighing from 500 to $750 \mathrm{~g}$. After purchase they were observed for at least $1 \mathrm{wk}$ while being allowed free access to water and food. Any animals appearing ill were excluded from the study.

Cells from along the crypt-villus axis were isolated using a modification of the technique described by Rowling and Sepulveda (3). After an overnight fast, rabbits were killed with an overdose of pentobarbital and the small bowel rapidly removed. After gently rinsing with warm PBS plus $1 \mathrm{mM}$ DTT, 20-cm segments were everted over a glass rod and filled with citrate buffer (in millimolar) $\mathrm{NaCl}, 96$; sodium citrate, $27 ; \mathrm{KH}_{2} \mathrm{PO}_{4}, 5.6 ; \mathrm{KCl}, 1.5$ adjusted to a $\mathrm{pH}$ of 7.3 . Both ends were tied and the loop placed in a $45-\mathrm{ml}$ tube containing citrate buffer. The intestine was incubated for $15 \mathrm{~min}$ at $37^{\circ} \mathrm{C}$ while being gently shaken. After this incubation, the loops were removed and placed in tubes containing isolation buffer (PBS plus $1.5 \mathrm{mM}$ EDTA and $0.5 \mathrm{mM}$ DTT) and incubated for timed intervals while undergoing gentle shak- 
ing. In this manner villus-tip cells were first eluted followed by midvillus cells. Cells were collected from the buffer by gentle centrifugation at $300 \mathrm{~g}$. Crypt cells were collected by gently scraping the remaining mucosa after both villus-tip and mid-villus cells were removed. After cell isolation, each fraction was pooled and microvillus membrane vesicles prepared as described by Kessler (4). For vesicle transport studies, vesicles were always prepared and used immediately. For lipid analysis, an aliquot of fresh membrane was immediately extracted by the method of Folch (5), dried under $\mathrm{N}_{2}$, and stored at $-70^{\circ} \mathrm{C}$ in chloroform. The remainder of the sample was frozen at $-70^{\circ} \mathrm{C}$ and used for enzymatic assays and determination of membrane fluidity.

Membrane analysis. All measurements were normalized to membrane protein content as determined by the method of Bradford (6). Sucrase activity was assessed in both the homogenate and microvillus membrane fraction by the method of Dalquist (7), and the thymidine kinase activity of the homogenate by the method of Klemperer (8). All lipid determinations were performed on samples extracted from fresh material. Total cholesterol was determined with an enzymatic method based on cholesterol oxidase (Boehringer Mannheim, Dorval, Quebec). All samples and standards were solubilized using $10 \%$ Triton in ethanol. Total phospholipids were determined by the method of Rouser (9).

To determine specific phospholipid subclasses, lipid extracts were spotted onto LK5D TLC plates (Whatman Inc., Clifton, NJ) that had been precoated with $1.2 \%$ boric acid in ethanol/water $(1: 1, \mathrm{vol} / \mathrm{vol})$ as described by Fine (10). Plates were developed with chloroform/methanol/water/ammonium hydroxide (120:75:6:2). The outside lanes of each plate were spotted with authentic standards and these were stained with iodine vapor at the end of the run, taking care not to stain the central, sample-containing lanes. Using these standards for identification, plates were carefully scored and $40 \mathrm{nmol}$ of pentadecanoic acid in hexane added to each phospholipid band as an internal standard. After drying under $\mathrm{N}_{2}$ the spots were aspirated into reaction tubes with a vacuum aspirator. Fatty acid methyl esters were prepared from the phospholipids using the method of Lepage and Roy (11). After extraction and drying under $\mathrm{N}_{2}$ the methyl esters were reconstituted in hexane and separated by gas-liquid chromatography. Separation was achieved with a $0.25-\mathrm{mm}$ capillary column (Supelcowax 10; Supelco, Inc., Bellefonte, PA) using a temperature-programmed run that clearly separated fatty acid methyl esters over the range of 14:0-26:0. Identification of each methyl ester was established from known standards and the use of mass-spectroscopy in selected cases. Using 15:0 as an internal standard, each phospholipid class could be quantitated by the method of Christie (12), and its fatty acid composition determined.

Fluidity determinations. Membrane fluidity was assessed using steady-state fluorescence polarization techniques and a variety of probes that allowed both the static and dynamic component of membrane fluidity to be estimated. The former was evaluated with the probe 1,6-diphenyl-1,3,5-hexatriene (DPH). ${ }^{1}$ Since the latter can be evaluated at several depths within the bilayer, a series of $n$-(9-anthroyloxy)-stearic or palmitic fatty-acid probes were used where $n$ took the values of $2,6,9,12$, and 16 . The techniques used for loading membranes with the probes and obtaining the experimental measurements have been previously described $(13,14)$. Data are reported as the steady-state anisotropy parameter. The term lipid fluidity is used to refer to the relative motional freedom of the lipid molecules in the bilayer. Using these probe molecules, an increase in fluidity corresponds to a decrease in either the rotational correlation time or the hindered anisotropy of the probe. For a further description of these terms and their implications see references 1,15 , or 16 .

1. Abbreviations used in this paper: DPH, 1,6-diphenyl-1,3,5-hexatriene; $J^{\text {max }}$, maximal glucose transport rate; $K_{\mathrm{m}}$, half-maximal rates of transport; PC, phosphatidylcholine; PE, phosphatidylethanolamine; $\mathrm{SPH}$, sphingomyelin.
Glucose transport. Vesicle uptake of D-glucose was assessed using a rapid filtration technique. Measurements were obtained over the concentration range of $10 \mu \mathrm{M}$ to $8 \mathrm{mM}$ using a 5-s time course. Preliminary experiments confirmed that uptake rates were linear over the first 8-10 s. Transport was initiated by rapidly mixing $10 \mu$ l of vesicles suspended in a vesicle buffer containing $250 \mathrm{mM}$ mannitol; $10 \mathrm{mM}$ Hepes; and $10 \mathrm{mM}$ Tris at pH 7.5 with $50 \mu \mathrm{l}$ of reaction buffer containing various concentrations of $\left[{ }^{3} \mathrm{H}\right]$ glucose, $50 \mathrm{mM}$ mannitol, 20 $\mathrm{mM}$ Hepes, and $100 \mathrm{mM} \mathrm{NaSCN}$ at $\mathrm{pH}$ 7.5. Sodium-independent rates of glucose transport were measured by replacing $\mathrm{NaSCN}$ with $\mathrm{KSCN}$. Transport was stopped by rapid dilution of the reaction mixture with $5 \mathrm{ml}$ of ice-cold stop solution containing $20 \mathrm{mM}$ Hepes; 100 $\mathrm{mM} \mathrm{NaCl}$; and $150 \mathrm{mM}$ mannitol at $\mathrm{pH}$ 7.5. The final solution was rapidly filtered through a $0.45-\mu \mathrm{m}$ filter (Millipore/Continental Water Systems, Bedford, MA), washed three times with stop solution, and counted in a liquid scintillation counter (Beckman Instruments, Inc., Palo Alto, CA). After quench correction the absolute amount of glucose associated with the vesicles was expressed as nanomole/minute per milligram protein. The data presented represent sodium-dependent glucose transport and were calculated by subtracting rates of uptake measured in the presence of a potassium gradient from total uptake. Concurrent with each experiment, vesicle volumes were measured by allowing vesicles to equilibrate with reaction solution over a 4-h period before filtration. The final size of the vesicles varied between 0.8 and $1.0 \mu \mathrm{l} / \mathrm{mg}$ protein and did not differ between groups.

Sodium transport. Rates of sodium influx were determined in microvillus membrane vesicles using the method of Rood et al. (17). Since these measurements were performed to estimate sodium-flux rates under conditions similar to those present during glucose transport experiments, they were performed in the absence of a $\mathrm{pH}$ gradient and amiloride. In preliminary experiments to validate this method it was found that, in our hands, rates of uptake were linear over the first $10 \mathrm{~s}$ of incubation. Thus, $30 \mu \mathrm{l}$ of vesicles was rapidly mixed with $60 \mu \mathrm{l}$ of buffer making a final cis sodium concentration of $1 \mathrm{mM}$ labeled with $1.2 \mu \mathrm{Ci}$ of $\mathrm{Na}^{22}$. After $5 \mathrm{~s}$ the reaction was stopped by the rapid addition of ice-cold stop solution, filtered and counted in a liquid scintillation counter (Beckman Instruments, Inc.). The buffers were used as described by Rood with the exception that Hepes was substituted for Mopso since the $\mathrm{pH}$ used for these studies was 7.5.

\section{Results}

Microvillus membrane characterization. The cell isolation technique used for these studies was evaluated by two major methods: histological analysis of the intestine and enzymatic characterization of the resulting cells and membrane preparations. Fig. 1 illustrates the removal of various portions of villus enterocytes by the shaking procedure outlined in Methods. As shown, shaking released villus enterocytes in a reproducible and progressive manner. By carefully timing the shaking procedure, we collected fractions enriched in villus-tip and midvillus cells, respectively. The appearance of the bowel after collection of villus-tip fractions is shown in panel $B$ of Fig. 1, while panels $C$ and $D$ illustrate the appearance after removal of the mid-villus fraction. It is clear that after collection of the villus-tip fraction both crypt and mid-villus enterocytes are still attached to the villi. After removal of the mid-villus cells the only enterocytes remaining are in the crypts that appear intact. These were removed by light scraping with a glass slide.

Enzymatic analysis is presented in Table I. Crypt cells were rich in activity of thymidine kinase and the activity of this enzyme rapidly dropped in the other fractions. Conversely, the activity of sucrase increased in the latter fractions confirming that these were more mature cells. As illustrated in Table I, sucrase activity was equally purified in microvillus membrane 

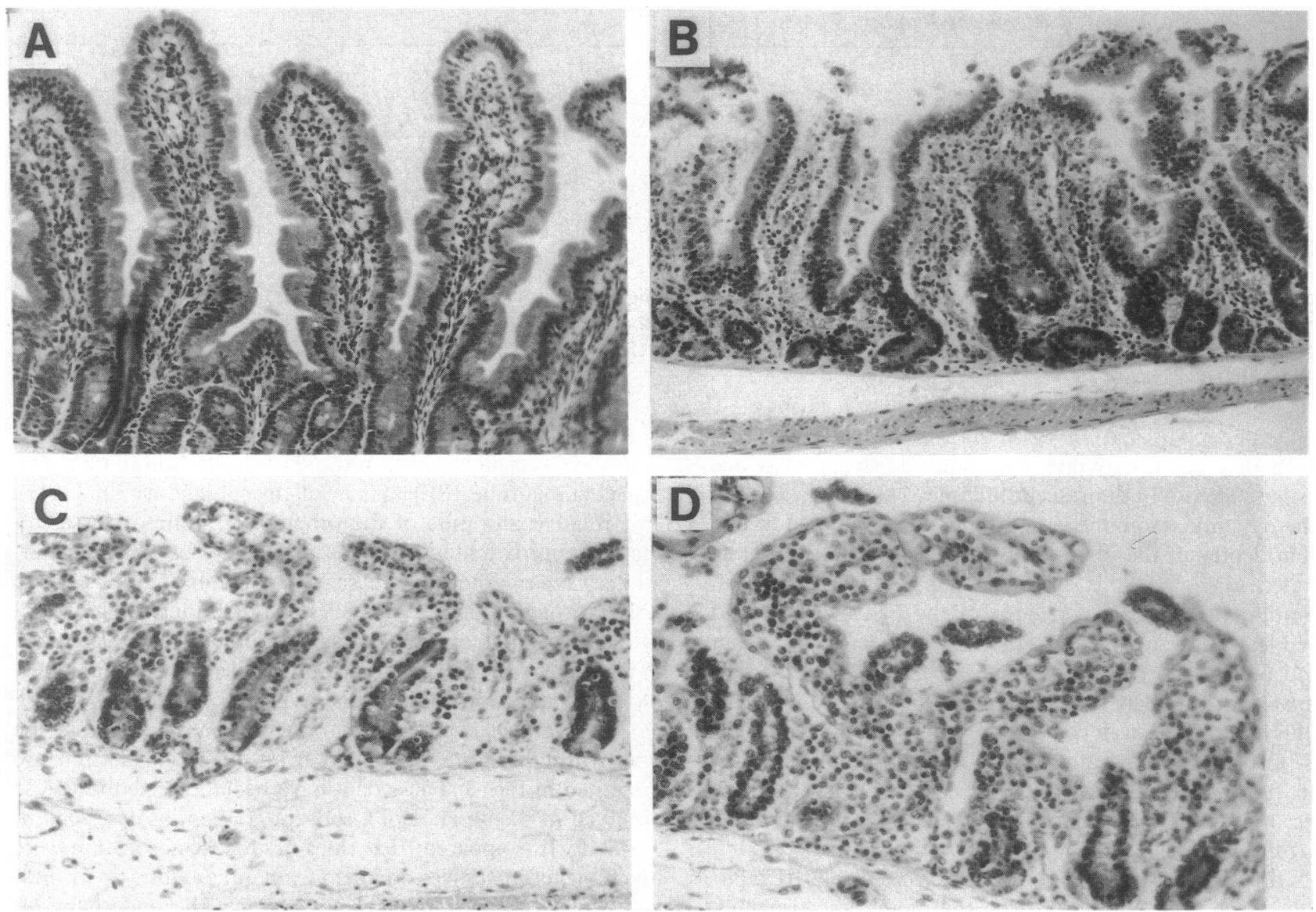

Figure 1. Cell isolation. $A$ is a representative section taken from the jejunum before cell isolation. Clearly seen are intact enterocytes along the entire crypt-villus axis. $B$ is a typical section taken at the conclusion of the shaking procedure used to collect villus-tip cells. As shown, only cells from the upper third of the villus have been removed. $C$ and $D$ are representative of the remaining intestine after removal of the mid-villus fraction. It can be clearly seen that the crypts remain intact after this isolation. They are subsequently removed by gentle scraping.

prepared from all three cell fractions despite the low activity of this enzyme in the crypt cell-enriched fractions. Although the apparent purification of crypt microvillus membrane was greater than the other fractions, this was not a statistically

Table I. Enzymatic Characterization of Cell Isolates

\begin{tabular}{lcccc}
\hline \multirow{2}{*}{$\begin{array}{c}\text { Membrane } \\
\text { source }\end{array}$} & $\begin{array}{c}\text { Homogenate } \\
\text { activity }\end{array}$ & $\begin{array}{c}\text { MVM } \\
\text { activity }\end{array}$ & $\begin{array}{c}\text { MVM } \\
\text { homogenate }\end{array}$ & $\begin{array}{c}\text { Thymidine } \\
\text { kinase }\end{array}$ \\
\cline { 2 - 5 } & U/mg protein & U/mg protein & -fold & $\begin{array}{c}\mu \text { mol/min } \\
\text { per } \text { mg protein }\end{array}$ \\
Crypt & $0.005 \pm 0.001^{*}$ & $0.2 \pm 0.1^{*}$ & $38.3 \pm 8.6$ & $11.8 \pm 1.2^{*}$ \\
Mid-villus & $0.058 \pm 0.004$ & $1.6 \pm 0.1$ & $27.4 \pm 2.3$ & $5.4 \pm 0.1$ \\
Villus-tip & $0.067 \pm 0.003$ & $1.6 \pm 0.1$ & $23.8 \pm 1.7$ & $3.9 \pm 2.1$ \\
& & & & \\
\hline
\end{tabular}

Values represent the mean \pm SEM for seven preparations from each membrane source. Thymidine kinase activity was assessed within homogenates rather than the final membrane pellet. The concentration of sucrase activity was calculated from the specific activity of this enzyme in both the homogenates and final pellet. MVM, microvillus membrane.

${ }^{*} P<0.001$ vs. both mid-villus and villus tip. significant difference. Significant contamination with basolateral membrane was excluded by the absence of detectable $\mathrm{Na}^{+}-\mathrm{K}^{+}$-ATPase activity in these membranes (data not shown).

Microvillus membrane physical properties. Using steadystate fluorescence polarization techniques, two components of membrane fluidity can be resolved. The static component of membrane fluidity can be assessed by probes such as DPH, while the dynamic component can be assessed with probes that undergo primarily rotational movements within the bilayer such as the anthroyloxy-labeled fatty acids (18). Inasmuch as the latter probes can have the fluorescent anthroyloxy group linked to any carbon of the base fatty acid, this property of the membrane can be evaluated as a function of depth within the bilayer $(19,20)$.

Table II presents data obtained with the probe DPH. Microvillus membrane obtained from crypt cells appeared significantly more fluid than that found in either villus-tip or midvillus cells. This is evident in both the anisotropy parameters and the calculated order parameter of the membrane. An alternative explanation for these data would be that the fluorescent lifetime of DPH increased in crypt microvillus membrane and produced the observed reduction in anisotropy parameters. However, fluorescent lifetime is proportional to fluorescent yield and, therefore, the decrease in total fluorescence 


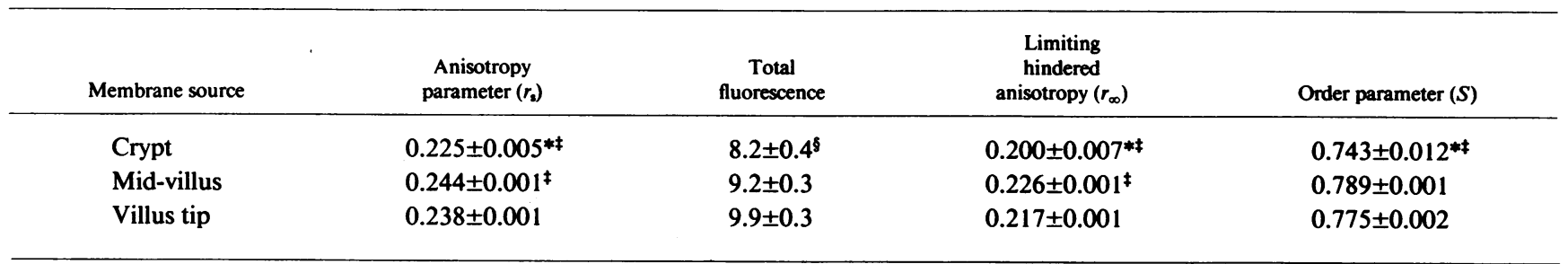

Values represent the mean \pm SEM for at least four determinations on seven membrane isolates for each group. Total fluorescence is reported in arbitrary fluorescence units for a constant amount of both membrane lipid and DPH. The calculation of both the limiting hindered anisotropy and the order parameter were performed according to methods outlined in reference 16 . $^{*} P<0.001$ vs. mid-villus, ${ }^{\ddagger} P<0.05$ vs. villus tip, $\S P<0.001$ vs. villus tip.

observed in crypt microvillus membrane is strong evidence against an increase in fluorescent lifetime.

The dynamic component of fluidity was evaluated at five different depths in the microvillus membrane and the results illustrated in Fig. 2. It is apparent that in each membrane preparation the superficial regions of the bilayer $(n=2-6)$ allowed less rotational freedom than the core, an observation previously reported $(1,13,14,21)$. However, distinct differences were observed between membrane isolates. Crypt microvillus membrane was consistently more fluid than either villus-tip or mid-villus microvillus membrane at all levels within the bilayer. Physical properties reported with these probes, however, were identical in villus-tip and mid-villus microvillus membrane.

Microvillus membrane lipid composition. Table III tabulates the basic lipid composition of the membranes isolated. Microvillus membrane obtained from crypt cells had more phospholipid per milligram of membrane protein resulting in a significantly reduced cholesterol/phospholipid ratio for these membranes. Furthermore, significant differences were noted in the phospholipid subclasses that formed these membranes. As the enterocyte matured and moved along the crypt-villus

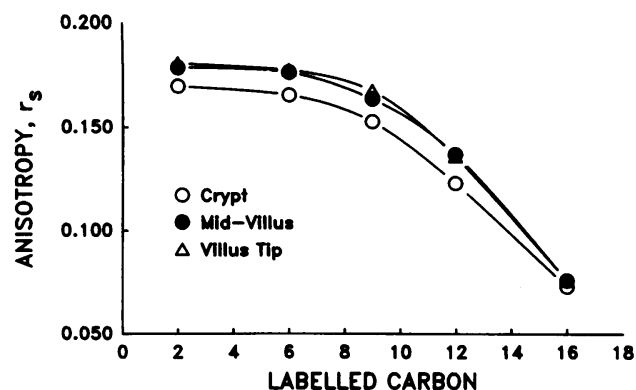

Figure 2. Dynamic component of membrane fluidity. Each point represents the mean of four determinations on each of seven membrane isolates for each source of membrane. The standard errors associated with each measurement are smaller than the point used to represent the mean. The vertical axis reflects the steady-state anisotropy parameter obtained using five different probes of the $n-(9$ anthroyloxy)-stearic or palmitic acid series. The numbers on the horizontal axis denote the value of $\boldsymbol{n}$ for each probe. Thus, for $\boldsymbol{n}=\mathbf{2}$ and 6 , superficial regions of the bilayer are being examined while for $n$ $=16$, physical properties of the core are being probed. For each and every value of $n$, anisotropy values obtained in crypt microvillus membrane were significantly different from those in mid-villus or villus-tip microvillus membrane. axis, there was an increase in the relative amount of phosphatidylethanolamine (PE) and a fall in sphingomyelin (SPH). The relative amounts of the other phospholipid classes remained approximately constant. The net result of these alterations was a significant increase in the $\mathrm{PE} /$ phosphatidylcholine (PC) ratio and a decrease in SPH/PC with increasing enterocyte maturity. Small but significant differences were also noted in the fatty-acid composition of microvillus membrane phospholipids along the crypt-villus axis (data not shown).

Glucose transport. Rates of glucose transport across the microvillus membrane isolated from each cell fraction are presented in Fig. 3. These data have been corrected for those observed in the absence of a sodium gradient as described in Methods. It is apparent that rates of glucose absorption were much higher in vesicles obtained from either villus-tip or midvillus than crypt enterocytes. To evaluate the underlying ki-

Table III. Lipid Composition

\begin{tabular}{lccc}
\hline & \multicolumn{3}{c}{ Membrane source } \\
\cline { 2 - 4 } \multicolumn{1}{c}{ Parameter } & \multicolumn{1}{c}{ Crypt } & Mid-villus & Villus tip \\
\hline $\begin{array}{l}\text { Cholesterol } \\
\quad \text { (nmol/mg protein) }\end{array}$ & $517 \pm 62$ & $610 \pm 16$ & $598 \pm 31$ \\
Total phospholipid & & & \\
$\quad($ nmol/mg protein) & $905 \pm 65^{*}$ & $754 \pm 22$ & $757 \pm 23$ \\
Cholesterol/phospholipid & & & \\
$\quad($ mol/mol) & $0.59 \pm 0.05^{*}$ & $0.86 \pm 0.03$ & $0.80 \pm 0.02$ \\
Lipid/protein $($ nmol/mg) & $1.42 \pm 0.2$ & $1.34 \pm 0.04$ & $1.35 \pm 0.05$ \\
Phospholipid species $(w t \%)$ & & & \\
PI & $12.4 \pm 2.6$ & $11.8 \pm 1.3$ & $9.0 \pm 1.1$ \\
PS & $11.0 \pm 1.5$ & $10.7 \pm 1.5$ & $10.9 \pm 0.7$ \\
SPH & $16.6 \pm 1.4^{*}$ & $8.9 \pm 0.9$ & $9.5 \pm 0.6$ \\
PC & $31.5 \pm 1.3$ & $31.4 \pm 1.0$ & $28.8 \pm 1.1$ \\
PE & $28.6 \pm 3.6^{*}$ & $37.2 \pm 1.5$ & $41.8 \pm 0.9$ \\
LPC & $<1$ & $<1$ & $<1$ \\
PE/PC $(w t / w t)$ & $0.91 \pm 0.10^{*}$ & $1.18 \pm 0.09$ & $1.45 \pm 0.09$ \\
SPH/PC $(w t / w t)$ & $0.53 \pm 0.12^{*}$ & $0.24 \pm 0.03$ & $0.23 \pm 0.02$ \\
\end{tabular}

Values represent the mean \pm SEM for determinations performed in triplicate on seven separate membrane isolates for each source of membrane. Abbreviations for phospholipid fractions are as follows; PI, phosphatidylinositol; PS, phosphatidylserine; SPH, sphingomyelin; PC, phosphatidylcholine; PE, phosphatidylethanolamine; LPC, lysophosphatidylcholine.

${ }^{*} P<0.01$ vs. both mid-villus and villus tip. 


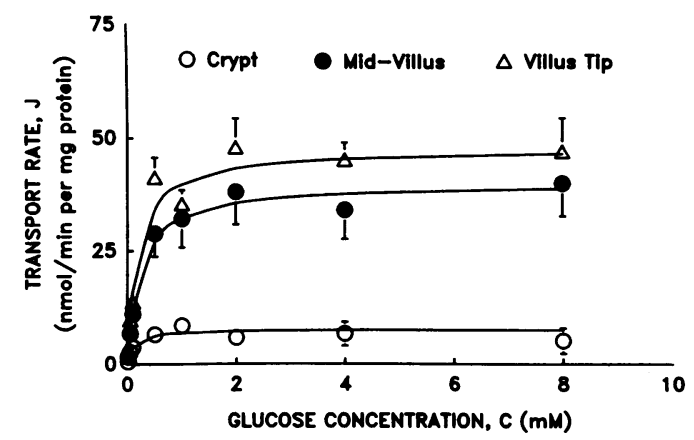

Figure 3. Glucose transport rates. Each data point represents the mean \pm SEM obtained in five membrane isolates from each region along the crypt-villus axis. In each isolate determinations were performed in quadruplicate for each concentration. The data shown represent the rate of glucose transport obtained in the presence of a 100 -mM inwardly directed sodium gradient minus that obtained with an inwardly directed $100 \mathrm{mM}$ potassium gradient. The vertical axis illustrates the rate of glucose transport, $J$, while the horizontal axis denotes the concentration at which the measurement was obtained. It is clear that crypt microvillus membrane is far less efficient at transporting glucose than either villus-tip or mid-villus microvillus membrane.

netic mechanisms for this difference these data were analyzed as described. In no case was it statistically necessary to postulate two kinetically distinct pathways to adequately fit these data. The resultant parameter estimates are presented in Table IV with the $95 \%$ confidence intervals for each parameter. As illustrated, microvillus membrane isolated from crypt enterocytes had a significantly lower maximal rate of glucose transport than found in microvillus membrane from either midvillus or villus-tip cells. The half-maximal rates of transport $\left(K_{\mathrm{m}}\right)$ for the transport system in the mature enterocyte was on the order of $200 \mu \mathrm{M}$. Surprisingly though, the $K_{\mathrm{m}}$ for the transport system in crypt microvillus membrane was almost twofold lower, $114 \mu \mathrm{M}$, a significant difference. Therefore, from a kinetic perspective, this may represent a different transporter.

To evaluate whether these differences in transport kinetics were related to the differences in membrane physical properties that we observed, an attempt was made to normalize these differences. In separate experiments using graded concentrations of benzyl alcohol, a recognized membrane fluidizer, we established that $50 \mathrm{mM}$ benzyl alcohol would increase the fluidity of villus-tip microvillus membrane, as assessed by DPH, to values observed in crypt microvillus membrane. Therefore, microvillus membrane was isolated from villus-tip enterocytes, after which the preparation was split into two aliquots. The first was incubated in $50 \mathrm{mM}$ methyl alcohol, to serve as an osmotic control, and the second in $50 \mathrm{mM}$ benzyl alcohol for $15 \mathrm{~min}$ before studying glucose transport.

Rates of glucose transport using these modified villus-tip microvillus membrane preparations are presented in Fig. 4, with the corresponding kinetic analysis presented in Table $\mathrm{V}$. This table also includes the observed steady-state anisotropy measurements obtained using DPH as a probe. A dramatic reduction in rates of glucose transport was observed in the fluidized villus-tip microvillus membrane (Fig. 4). Maximal rates of glucose transport fell by a factor of 4 , becoming indistinguishable from values observed in native crypt microvillus membrane. Furthermore, the $K_{\mathrm{m}}$ for the transport process also decreased significantly from 198.3 to $104 \mu \mathrm{M}$. Kinetically, these vesicles appeared to be crypt microvillus membrane, and as illustrated in Table $\mathrm{V}$, the microenvironment assessed by DPH was also cryptlike. The addition of methyl alcohol did not significantly alter either the physical properties or kinetic parameters of the vesicles.

These data raised several important questions to us. Firstly, the observed reduction in $K_{\mathrm{m}}$ associated with increasing fluidity was intriguing in view of observations reported by Wright et al. (22). These authors recently reported that the $K_{\mathrm{m}}$ of the transporter is sensitive to the cis sodium concentration, being on the order of $200 \mu \mathrm{M}$ when the cis sodium was $25 \mathrm{mM}$ and as this concentration was increased to $100 \mathrm{mM}$ the $K_{\mathrm{m}}$ fell to $\sim 100 \mu \mathrm{M}$. The interpretation offered by these authors was that initial binding of sodium to the transporter initiated a conformational change in the transporter that increased its affinity for glucose. Our values for the $K_{\mathrm{m}}$ in crypt vesicles are similar to these but in the more mature enterocytes the $K_{\mathrm{m}}$ remained close to $200 \mu \mathrm{M}$, despite the presence of a $100-\mathrm{mM}$ cis-sodium gradient. This might suggest that two transporters are present or, alternatively, that the conformational change induced by sodium binding can only occur in a membrane possessing a certain minimal fluidity. Under this interpretation we would suggest that mid-villus and villus-tip microvillus membrane is too rigid to allow this change but that it occurred in the crypt vesicles. To test this hypothesis we analyzed the kinetics of glucose transport in crypt vesicles over the same
Table IV. Kinetic Parameters Describing Glucose Transport

\begin{tabular}{|c|c|c|c|c|}
\hline \multirow[b]{2}{*}{ Location } & \multicolumn{2}{|r|}{$J^{\max }$} & \multicolumn{2}{|r|}{$K_{\mathrm{m}}$} \\
\hline & Estimate & $\begin{array}{l}\text { 95\% Confidence } \\
\text { intervals }\end{array}$ & Estimate & $\begin{array}{l}\text { 95\% Confidence } \\
\text { intervals }\end{array}$ \\
\hline & \multicolumn{2}{|c|}{ nmol/min per mg protein } & \multicolumn{2}{|r|}{$\mu M$} \\
\hline Crypt & $7.68^{* \neq}$ & $6.13,9.23$ & $114.3^{* \ddagger}$ & $75.3,153.3$ \\
\hline Mid-villus & 40.03 & $33.92,46.14$ & 242.4 & $180.6,304.2$ \\
\hline Villus tip & 47.75 & $42.38,53.11$ & 200.4 & $157.8,242.9$ \\
\hline
\end{tabular}

Values represent the best parameter estimate and its associated $95 \%$ confidence interval for the experiment illustrated in Fig. 3. Data were obtained from nonlinear regression analysis as described. ${ }^{*} P<0.0001$ vs. mid-villus, ${ }^{\ddagger} P<0.001$ vs. villus tip.

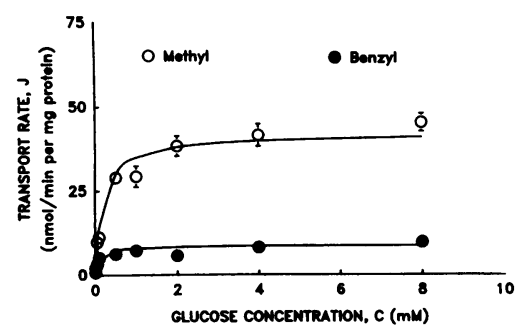

Figure 4. Effect of membrane fluidization on glucose transport rates. The data shown were obtained from four membrane isolates different than those used in Fig. 3. As discussed in the text, villus-tip microvillus

membrane was isolated and allowed to incubate with either $50 \mathrm{mM}$ benzyl alcohol (fluidizer) or methyl alcohol (osmotic control). Otherwise the format of the figure is identical to that of Fig. 3. Fluidized vesicles are illustrated in filled circles while the controls are shown in open circles. It is clear that the addition of benzyl alcohol had a profound effect on rates of glucose transport, an observation that is discussed more completely in the text. 


\begin{tabular}{|c|c|c|c|c|c|c|}
\hline \multirow[b]{2}{*}{ Sample } & \multicolumn{2}{|c|}{$J^{\max }$} & \multicolumn{2}{|c|}{$K_{\mathrm{m}}$} & \multirow[b]{2}{*}{$\begin{array}{c}\text { Anisotropy } \\
\text { parameter }\left(r_{\mathrm{s}}\right)\end{array}$} & \multirow[b]{2}{*}{$\begin{array}{l}\text { Order parameter } \\
\qquad(S)\end{array}$} \\
\hline & Estimate & $\begin{array}{l}\text { 95\% Confidence } \\
\text { intervals }\end{array}$ & Estimate & $\begin{array}{l}95 \% \text { Confidence } \\
\text { intervals }\end{array}$ & & \\
\hline & \multicolumn{2}{|c|}{$\mathrm{nmol} / \mathrm{min}$ per $\mathrm{mg}$ protein } & \multicolumn{2}{|c|}{$\mu M$} & & \\
\hline Methyl alcohol & 42.0 & $38.7,45.2$ & 198.3 & $162.9,233.6$ & $0.240 \pm 0.001$ & $0.780 \pm 0.002$ \\
\hline Benzyl alcohol & $8.8^{*}$ & $7.1,10.5$ & $104.2^{\ddagger}$ & $60.5,147.8$ & $0.220 \pm 0.001^{*}$ & $0.731 \pm 0.001^{*}$ \\
\hline
\end{tabular}

Values represent the mean \pm SEM or $95 \%$ confidence intervals for the experiments illustrated in Fig. $4 . \quad * P<0.0001$ vs. methyl alcohol, ${ }^{\ddagger} P<0.05$ vs. methyl alcohol.

glucose concentration range but with a cis sodium concentration of $25 \mathrm{mM}$. In three separate crypt-cell preparations the $K_{\mathrm{m}}$ for this transport system under these conditions was $223.6 \pm 23.3$. These data support the conclusion that the conformational change induced by sodium can only be expressed in relatively fluid microvillus membrane.

Secondly, the observed reduction in maximal glucose transport rate $\left(J^{\max }\right)$ associated with membrane fluidization was dramatic and we were concerned that this might represent an effect of benzyl alcohol unrelated to increasing membrane fluidity. We proceeded to investigate this by fluidizing villustip vesicles with another alcohol, hexanol. Using DPH as a marker, similar degrees of fluidization could be obtained by incubating vesicles with $15 \mathrm{mM}$ hexanol for $15 \mathrm{~min}$. The kinetic parameters for glucose transport in five separate membrane preparations treated in this manner are also reported in Table V. The cis sodium concentration for these experiments was fixed at $100 \mathrm{mM}$. Despite a similar increase in fluidity, measured with DPH, the reduction in $J^{\max }$ was significant but not of the same order of magnitude as seen with $50 \mathrm{mM}$ benzyl alcohol. Two possible explanations occurred to us. First, the pattern of increasing fluidity induced by hexanol might be different from that of benzyl alcohol, thereby having a different effect on the transporter. In order to address this concern we carefully analyzed the fluidity properties of villus-tip microvillus membrane treated with either methanol, hexanol, or benzyl alcohol. These data are presented in Table VI and represent five separate villus-tip microvillus membrane preparations prepared and incubated in the alcohols shown. Of im- portance is the observation that hexanol made villus-tip microvillus membrane even more fluid than did benzyl alcohol no matter which probe was used for the comparison. Therefore, the differential reduction in $J^{\max }$ induced by hexanol and benzyl alcohol could not be explained by a more significant fluidization of these membranes with benzyl alcohol.

The other possibility was that the dramatic effect of benzyl alcohol on rates of glucose transport was not entirely due to its effect on the glucose transporter. A similar result would have been anticipated if benzyl alcohol increased sodium permeability of the vesicles and thereby collapsed the sodium gradient across the microvillus membrane. In order to examine this possibility, villus-tip vesicles were prepared as described but incubated in either methanol, hexanol, or benzyl alcohol before measuring $\mathrm{Na}^{+}$permeability. Inside and outside $\mathrm{pH}$ was fixed at 7.5. A final cis sodium concentration of $1 \mathrm{mM}$ was employed with $1.2 \mu \mathrm{Ci}$ of $\mathrm{Na}^{22}$ added to the buffer in the absence of glucose. Inasmuch as amiloride was not used in the glucose transport assays, this was not included for these experiments. In preliminary time courses (data not shown) it was demonstrated that a 5-s incubation was within the linear portion of the assay and, therefore, this incubation time was used for subsequent experiments. Rates of sodium movement into villus-tip vesicles, under these conditions, are shown in Table VII. It is apparent that no differences were observed between control vesicles or those treated with either methanol or hexanol. However, villus-tip microvillus membrane vesicles treated with benzyl alcohol had significantly increased rates of sodium entry into the vesicle.

Table VI. Fluidity of Villus-Tip Microvillus Membrane in the Presence of Different Alcohols

\begin{tabular}{lcccr}
\hline & \multicolumn{3}{c}{ Anisotropy parameter } \\
\cline { 2 - 5 } Probe & None & Methanol & Benzyl & Hexanol \\
\hline 2-AS & $0.176 \pm 0.002$ & $0.182 \pm 0.002$ & $0.171 \pm 0.001$ & $0.164 \pm 0.002$ \\
6-AS & $0.172 \pm 0.002$ & $0.180 \pm 0.002$ & $0.173 \pm 0.001$ & $0.163 \pm 0.001$ \\
9-AS & $0.165 \pm 0.001$ & $0.167 \pm 0.002$ & $0.155 \pm 0.001$ & $0.148 \pm 0.001$ \\
12-AS & $0.137 \pm 0.001$ & $0.140 \pm 0.001$ & $0.126 \pm 0.001$ & $0.117 \pm 0.001$ \\
16-AP & $0.083 \pm 0.001$ & $0.084 \pm 0.001$ & $0.080 \pm 0.001$ & $0.072 \pm 0.001$ \\
DPH & $0.246 \pm 0.005$ & $0.247 \pm 0.002$ & $0.229 \pm 0.001$ & $0.224 \pm 0.001$
\end{tabular}

Data represent the steady-state anisotropy parameter for the probes listed obtained in four separate membrane preparations incubated with the alcohol listed. The concentrations for each alcohol (in millimolar) were methanol, 50; benzyl, 50; and hexanol, 15 . Each value obtained for the hexanol-treated membranes was significantly lower than that observed in either the control or benzyl-treated group. No differences in fluorescent lifetime were observed. 
Table VII. Sodium-Flux Rates in Fluidized Villus-Tip Vesicles

\begin{tabular}{lc}
\hline \multicolumn{1}{c}{ Fluidizer } & $J_{\mathrm{Na}}$ \\
\hline & $n$ mol/min per $m$ m protein \\
None & $3.9 \pm 2.4$ \\
Methyl alcohol (50) & $4.2 \pm 2.1$ \\
Hexanol (15) & $3.8 \pm 2.7$ \\
Benzyl alcohol (50) & $21.5 \pm 3.5^{*}$
\end{tabular}

The concentration of each fluidizer, in millimolar, is given in parentheses.

* $P=0.002$ vs. control or methyl alcohol-treated vesicles.

\section{Discussion}

The enterocyte lives a relatively short life. After inception in the crypt it is pushed up onto the villus by pressure from newly formed crypt enterocytes and in 3-4 d reaches the tip of the villus where it is subsequently sloughed into the intestinal stream. Despite its short life span the enterocyte becomes a polarized cell with a tremendous array of transport and metabolic systems. It has recently become clear that many of these processes are differentially expressed along the crypt-villus axis, presumably related to cell maturity. The present study confirms these observations for microvillus membrane glucose transport, lipid composition, and membrane physical properties in the rabbit.

Microvillus membrane isolated from crypt enterocytes was far more fluid than microvillus membrane isolated from either mid-villus or villus-tip cells in terms of both the static and dynamic component of membrane fluidity. Furthermore, for the latter these differences persisted at all depths within the bilayer from the superficial regions to the core. When using steady-state techniques to measure membrane fluidity, it is important to appreciate that the fluorescent lifetime of the probe contributes to the experimentally measured anisotropy parameters. Therefore, it is conceivable that what appears to be an increase in membrane fluidity is in reality only a prolonged fluorescent lifetime of the probe. However, since total fluorescence of the sample is directly proportional to fluorescent lifetime, this parameter should increase if lifetime increases. In this study we have demonstrated that crypt microvillus membrane appeared to be not only more fluid than villus-tip and mid-villus membranes but total fluorescence was decreased, making it difficult to implicate a change in fluorescent lifetime to account for this difference. Therefore, we conclude that the crypt microvillus membrane environments sampled by the probes used in this study are radically different from those encountered in more mature enterocytes. Similar findings were recently reported by Brasitus and Dudeja in the rat (1) where direct measurements of fluorescent lifetimes were made. These investigators found that the microvillus membrane was more fluid in crypt cells and, furthermore, that fluorescent lifetimes of the probes actually decreased in these immature membranes. This is entirely consistent with our finding of decreased total fluorescence in crypt membranes and would suggest that we have in fact underestimated the difference between crypt and villus-tip microvillus membrane. As the rat enterocyte matured, the cholesterol/phospholipid ratio, saturated fatty acids, and the protein/lipid ratio all in- creased in the study reported by Brasitus (1). All of these observations helped to explain the reduction in microvillus membrane fluidity associated with maturation of the rat enterocyte. Similar findings were observed in our study. However, in the rabbit we observed an increase in the relative amount of PE, a change also associated with reductions in membrane fluidity (23). Curiously, we were unable to confirm an increase in phospholipid acyl saturation with increasing maturity in the rabbit. In fact, for virtually all phospholipid subclasses we found the opposite. With increasing maturity of the microvillus membrane the relative weight percentage of saturated fatty acids fell and they were replaced by polyunsaturated fatty-acyl chains, the monounsaturated fraction remaining relatively constant. We also observed a significant decrease in SPH content with increasing maturity, a change that would predispose to a more fluid, not more rigid, membrane with increasing maturity. However, it is apparent that this alteration was more than compensated for by the increase in cholesterol/phospholipid ratio and $\mathrm{PE} / \mathrm{PC}$ ratio. A similar trend was documented in the rat (1) but the differences were not significant.

It has been recognized that the intestinal crypts do not contribute significantly to the transport of a variety of nutrients. Whether this is related to a lack of transporters, decreased transporter function, lack of metabolizing enzymes, or even the inability of nutrients to diffuse into the crypt region is unclear. To our knowledge no other kinetic studies of glucose transport have been previously reported in microvillus membrane vesicles isolated from along the crypt-villus axis. A recent study by Knickelbein (24) examined electrolyte exchange across similar vesicles. In this report a brief mention is made suggesting that sodium-dependent glucose transport was reduced in crypt microvillus membrane, but without kinetic studies no attempt was made to speculate on the mechanism. Using piglets suffering from gastroenteritis, Keljo et al. (25) reported hypertrophy of crypt cells. Vesicles prepared from this crypt-enriched material demonstrated a reduction in the rate of glucose transport. From kinetic studies the authors concluded that vesicles from crypt-enriched fractions had only a low affinity transporter and that vesicles from controls (villus plus crypt cells) had both a high and low affinity transport system.

Our data do not entirely support these observations. Like Keljo et al. (25) we found glucose transport to be markedly depressed in crypt microvillus membrane vesicles. However, in no fraction was there convincing kinetic evidence for a dual transport system. Kinetic analysis of our data revealed that both mid-villus and villus-tip microvillus membrane had a single sodium-dependent glucose transporter with an affinity for glucose of $\sim 200 \mu \mathrm{M}$. Microvillus membrane isolated from crypt cells had a much lower $J^{\text {max }}$, but this transport system had an affinity for glucose almost twice that found in the more mature enterocytes. One possible interpretation is that immature cells have one form of the sodium-dependent glucose transporter (high affinity, low capacity) and as the cell matures a second transporter, the adult form (low affinity, high capacity), becomes evident and takes over. However, if this explanation is correct our data would suggest that by the time the enterocyte reaches the lower region of the villus, the immature, high affinity transporter is completely replaced by the adult transporter since we do not find evidence for two transporters in the mid-villus fraction. Although appealing, it seems un- 
likely that an intermediate condition would not exist and that the emergence of the adult form of the transporter was complete in such a rapid time.

A second interpretation was suggested by the observation that the difference in transporter affinity correlated so well with the alteration in membrane physical properties. Both the mid-villus and villus-tip microvillus membrane were significantly less fluid than crypt microvillus membrane and expressed a glucose transporter with a lower affinity for glucose than found in more fluid microvillus membrane. It is conceivable that the same glucose transporter exists in all three membrane fractions but expresses itself differently in different lipid environments. This question was addressed by preparing villus-tip microvillus membrane and artificially fluidizing it to levels seen in the crypt microvillus membrane. When glucose transport rates were determined in fluidized preparations, they uniformly decreased. Kinetic analysis revealed reductions in $J^{\max }$, and an increase in the affinity of the transport system to levels indistinguishable from those found in native crypt microvillus membrane. This provided support for the latter interpretation and is not without precedent. Recent work has suggested that the sodium-dependent glucose transport system may be modulated by alterations in the lipid fluidity of the membrane they are embedded in. By inducing reversible renal ischemia Molitoris and Kinne (26) have shown alterations in the microvillus membrane of the proximal tubule that result in a more fluid lipid environment than controls. While rates of alanine transport were unaffected, fluidization of the vesicle membrane was associated with a dramatic reduction in maximal rates of sodium-dependent glucose transport. Furthermore, the activity of the sodium-independent glucose transporter from both the adipocyte and fibroblast have been shown to be amenable to alterations in the membrane lipid environment (27-29).

However, the relationship between membrane fluidity and sodium-dependent glucose transport is still problematical. Fluidizing microvillus membrane vesicles had two major effects on the sodium-dependent glucose transport system. Firstly, it allowed for an efficient conformational change in the protein induced by sodium binding. In the presence of a cis sodium concentration of $100 \mathrm{mM}$, relatively rigid villus-tip vesicles have a $K_{\mathrm{m}}$ close to $200 \mu \mathrm{M}$ while relatively fluid crypt vesicles have a $K_{\mathrm{m}}$ closer to $100 \mu \mathrm{M}$. Fluidizing villus-tip vesicles with benzyl alcohol increases the affinity of the transporter to values indistinguishable from those observed in native crypt microvillus membrane. Recent work by Wright et al. (22) has suggested that the binding of sodium induces a conformational change in the transporter that increases its affinity for glucose. By reducing the cis sodium concentration to $25 \mathrm{mM}$ we were able to demonstrate that the $K_{\mathrm{m}}$ for the transporter in crypt microvillus membrane increased to values close to $200 \mu \mathrm{M}$, similar to those found by Wright (22) and in our villus-tip vesicles. This would suggest that the postulated conformational change associated with sodium binding can only take place if the surrounding membrane has a certain minimal fluidity. This level of fluidity seemed to be present in crypt microvillus membrane and fluidized villus-tip vesicles but not in native, rigid villus-tip microvillus membrane. Thus, it appears that the first and foremost effect of fluidizing microvillus membrane is to allow an efficient conformational change of the glucose transporter upon sodium binding.

The second effect of fluidization was to reduce maximal rates of glucose transport. However, the magnitude of this effect was dramatically different for the two membrane fluidizers, hexanol and benzyl alcohol. These differences could not be explained by differences in membrane fluidity induced by these two alcohols. Hexanol, at concentrations used in this study, fluidized microvillus membrane even more efficiently than benzyl alcohol. While hexanol significantly decreased maximal rates of glucose transport these reductions did not approach the magnitude of those seen with benzyl alcohol. Thus, a second explanation for the dramatic effect of benzyl alcohol was looked for.

It is technically difficult to examine rates of sodium permeation across the microvillus membrane under the conditions used to measure glucose transport (a 100-mM cis sodium concentration), and therefore, sodium movement into villus-tip vesicles was examined at a cis sodium concentration of 1 $\mathrm{mM}$. Under these conditions it was apparent that benzyl alcohol, in the concentrations employed to fluidize villus-tip vesicles, induced a significantly greater rate of sodium flux into microvillus membrane vesicles than did hexanol. Thus, the use of benzyl alcohol to fluidize membranes in this study, and possibly in others, overestimated the effect of membrane fluidization on rates of sodium-dependent glucose transport as it increased sodium flux by other mechanisms. The nature of this mechanism is interesting since hexanol, which had a greater effect on membrane fluidity, did not alter rates of sodium flux. However, despite these observations, fluidizing villus-tip microvillus membrane with hexanol significantly reduced the maximal rate of glucose transport without a measurable effect upon either sodium permeability or vesicular size.

In summary, we have confirmed the observations of Brasitus and Dudeja (1), made in the rat, that the microvillus membrane undergoes a process of maturation in terms of its physical properties with migration of the enterocyte up the crypt-villus axis. Furthermore, we have identified the underlying alterations in lipid composition that accompany this change and presumably account for it. Moreover, we have characterized sodium-dependent glucose transport into these vesicles in kinetic terms and established that not only is glucose transport inefficient in the crypts of intact animals but that this observation also holds true for movement across the microvillus membrane. Therefore, at least part of the reason that glucose is poorly absorbed in the crypt region may be ascribed to the lack of functional transporters in crypt microvillus membrane. We were able to characterize two different forms of the glucose transporter in terms of its $K_{\mathrm{m}}$. However, it appeared that the expression of $K_{\mathrm{m}}$ was dependent upon the physical properties of the membrane in which it was embedded. Fluidization of membranes, in vitro, decreased both the $J^{\max }$ and $K_{\mathrm{m}}$ of the glucose transport system, however, this effect was not sufficient to account for the differences in $J^{\max }$ observed between crypt and villus-tip microvillus membrane. Thus, differences in membrane physical properties fail to entirely explain markedly suppressed rates of glucose transport across crypt as compared with villus-tip microvillus membrane. It would appear that crypt microvillus membrane has fewer transporters than villus-tip microvillus membrane, however, in the rabbit these transporters are kinetically similar.

\section{Acknowledgments}

The authors wish to acknowledge the care and patience devoted to the preparation of this manuscript by I. McGovern. 
These studies were supported by grants from the Alberta Heritage Foundation for Medical Research and the Medical Research Council of Canada.

\section{References}

1. Brasitus, T. A., and P. K. Dudeja. 1985. Alterations in the physical state and composition of brush border membrane lipids of rat enterocytes during differentiation. Arch. Biochem. Biophys. 240:483488.

2. Meddings, J. B., R. B. Scott, and G. H. Fick. 1990. Analysis and comparison of sigmoidal curves: application to dose-response data. Am. J. Physiol. 257:G982-G989.

3. Rowling, P. J. E., and F. V. Sepulveda. 1984. The distribution of $\left(\mathrm{Na}^{+}+\mathrm{K}^{+}\right)$-ATPase along the villus crypt-axis in the rabbit small intestine. Biochim. Biophys. Acta. 771:35-41.

4. Kessler, M., O. Acuto, C. Storelli, H. Murer, M. Muller, and G. Semenza. 1978. A modified procedure for the rapid preparation of efficiently transporting vesicles from small intestinal brush border membranes. Their use in investigating some properties of D-glucose and choline transport systems. Biochim. Biophys. Acta. 506:136-154.

5. Folch, J., M. Lees, and G. H. Sloane. 1957. A simple method for isolation and purification of total lipids from animal tissues. J. Biol. Chem. 226:497-509.

6. Bradford, M. M. 1976. A rapid and sensitive method for the quantitation of microgram quantities of protein utilizing the principle of protein-dye binding. Anal. Biochem. 72:248-254.

7. Dalquist, A. 1964. Method for assay of intestinal disaccharidases. Anal. Biochem. 7:18-25.

8. Klemperer, H. G., and G. R. Haynes. 1968. Thymidine kinase in rat liver during development. Biochem. J. 108:541-546.

9. Rouser, G., S. Fleischer, and A. Yamamoto. 1970. Two dimensional thin layer chromatographic separation of polar lipids and determination of phospholipids by phosphorous analysis of spots. Lipids. 5:494-496.

10. Fine, J. B., and H. Sprecher. 1982. Unidimensional thin-layer chromatography of phospholipids on boric acid-impregnated plates. $J$. Lipid Res. 23:660-663.

11. Lepage, G., and C. C. Roy. 1986. Direct transesterification of all classes of lipids in a one-step reaction. J. Lipid Res. 27:114-120.

12. Christie, W. W., R. C. Noble, and J. H. Moore. 1970. Determination of lipid classes by a gaschromatographic procedure. Analyst. 95:940-944.

13. Meddings, J. B., and S. Theisen. 1989. Development of the rat jejunum: lipid permeability, physical properties and chemical composition. Am. J. Physiol. 256:G931-G940.

14. Meddings, J. B. 1988. Lipid permeability of rat jejunum and ileum: correlation with physical properties of the microvillus membrane. Biochim. Biophys. Acta. 943:305-314.

15. Brasitus, T. A., R. Dahiya, P. K. Dudeja, and B. M. Bisson- nette. 1988. Cholesterol modulates alkaline phosphatase activity of rat intestinal microvillus membranes. J. Biol. Chem. 263:8592-8597.

16. van Blitterswijk, W. J., R. P. van Hoeven, and B. W. van der Meer. 1981. Lipid structural order parameters (reciprocal of fluidity) in biomembranes derived from steady-state fluorescence polarization measurements. Biochim. Biophys. Acta. 644:323-332.

17. Rood, R. P., E. Emmer, J. Wesolek, J. McCullen, Z. Husain, M. E. Cohen, R. S. Braithwaite, H. Murer, G. W. G. Sharp, and M Donowitz. 1988. Regulation of the rabbit ileal brush-border $\mathrm{Na}^{+} / \mathrm{H}^{+}$ exchanger by an ATP-requiring $\mathrm{Ca}^{++} /$calmodulin-mediated process. $J$. Clin. Invest. 82:1091-1097.

18. Molitoris, B. A. 1987. Membrane fluidity: measurement and relationship to solute transport. Semin. Nephrol. 7:61-71.

19. Thulborn, K. R., and W. H. Sawyer. 1978. Properties and the locations of a set of fluorescent probes sensitive to the fluidity gradient of the lipid bilayer. Biochim. Biophys. Acta. 511:125-140.

20. Thulborn, K. R. 1981. The use of $n$-(9-anthroyloxy) fatty acids as fluorescent probes for biomembranes. In Fluorescent Probes. G. S. Beddard and M. A. West, editors. Academic Press Limited, London. 113-141.

21. Schachter, D., and M. Shinitzky. 1977. Fluorescence polarization studies of rat intestinal microvillus membranes. J. Clin. Invest 59:536-548.

22. Peerce, B. E., and E. M. Wright. 1984. Sodium-induced conformational changes in the glucose transporter of intestinal brush borders. J. Biol. Chem. 259:14105-14112.

23. van Blitterswijk, W. J., B. W. van der Meer, and H. Hilkmann. 1987. Quantitative contributions of cholesterol and the individual classes of phospholipids and their degree of fatty acyl (un)saturation to membrane fluidity measured by fluorescence polarization. Biochemistry. 26:1746-1756.

24. Knickelbein, R. G., P. S. Aronson, and J. W. Dobbins. 1988. Membrane distribution of sodium-hydrogen and chloride-bicarbonate exchangers in crypt and villus cell membranes from rabbit ileum. $J$. Clin. Invest. 82:2158-2163.

25. Keljo, D. J., R. J. Macleod, M. H. Perdue, D. G. Butler, and J. R. Hamilton. 1985. D-glucose transport in piglet jejunal brush border membranes: insights from a disease model. Am. J. Physiol. 249:G751-G760.

26. Molitoris, B. A., and R. Kinne. 1987. Ischemia induces surface membrane dysfunction. Mechanism of altered $\mathrm{Na}^{+}$-dependent glucose transport. J. Clin. Invest. 80:647-654.

27. Melchiro, D. L., and M. P. Czech. 1979. Sensitivity of the adipocyte D-glucose transport system to membrane fluidity in reconstituted vesicles. J. Biol. Chem. 254:8744-8747.

28. Pilch, P. F., P. A. Thompson, and M. P. Czech. 1980. Coordinate modulation of D-glucose transport activity and bilayer fluidity in plasma membranes derived from control and insulin-treated adipocytes. Proc. Natl. Acad. Sci. USA. 77:915-918.

29. Yuli, I., W. Wilbrandt, and M. Shinitzky. 1981. Glucose transport through cell membranes of modified lipid fluidity. Biochemistry. $20: 4250-4256$. 